\title{
The Undersampled Discrete Gabor Transform
}

\author{
Sigang Qiu
}

\begin{abstract}
Conventional studies on discrete Gabor transforms have generally been confined to the cases of critical sampling and oversampling in which the Gabor families span the whole signal space.

In this paper, we investigate undersampled discrete Gabor transforms. For an undersampled Gabor triple $(g, a, b)$, i.e., $a \cdot b>N$, we show that the associated generalized dual Gabor window (GDGW) function is the same as the one associated with the oversampled $(\boldsymbol{g}, N / b, N / d)$, except for the constant factor $(a b / N)$. Computations of undersampled Gabor transforms are made possible. By applying the methods (algorithms) developed in oversampled settings, the undersampled GDGW is determined. Then, we are able to obtain the best approximation of a signal $x$ by linear combinations of vectors in the Gabor family.
\end{abstract}

Index Terms-Gabor operator, Gabor transform, generalized dual Gabor window (GDGW).

\section{INTRODUCTION AND PRELIMINARIES}

$\mathbf{T}$ WHE SUBJECT of the Gabor transform [12] concerns the problem of representing signals in terms of building blocks that form a Gabor family. The Gabor family is obtained from one single function by appropriate time and frequency shifts. For a signal $f(t)$, the Gabor representation of $f(t)$ is defined as

$$
f(t)=\sum_{n, m} C_{n, m} h_{n, m}(t)
$$

where

$$
h_{n, m}=h(t-n T) e^{-2 \pi i m \Omega t}
$$

$i=\sqrt{-1}, h(t)$ is a Gabor (synthesis) window function, and $\left\{C_{n, m}\right\}$ is a set of Gabor coefficients.

In order to do digital computation for the Gabor transforms, the authors in [1], [16], [28], [30], and others have derived discrete formulations of (1). In this paper, we study the discrete Gabor transform (DGT). In order to briefly review the DGT, we need to set up relevant notation.

\section{A. Notation}

1) Let $N$ be a fixed positive integer. A signal $x$ is represented as a row vector $\boldsymbol{x}=\{x(k)\}_{k=0}^{N-1} \in \mathbb{C}^{N}$. Throughout the paper, $x$ is assumed to be $N$ periodic, i.e., $\boldsymbol{x}(j+k N)=\boldsymbol{x}(j)$ for $j=0,1, \cdots, N-1$ and $k \in \mathbb{Z}$. The inner product of two signals is expressed as $\langle\boldsymbol{x}, \boldsymbol{y}\rangle=\sum_{j=0}^{N-1} x(j) \overline{y(j)}$. $\|\boldsymbol{x}\|:=\langle\boldsymbol{x}, \boldsymbol{x}\rangle^{1 / 2}$ is the

Manuscript received August 3, 1995; revised June 19, 1997. The associate editor coordinating the review of this paper and approving it for publication was Dr. Nurgun Erdol.

The author is with Cirrus Logic, Raleigh, NC 27615 USA.

Publisher Item Identifier S 1053-587X(98)03178-X
Euclidean norm of $\boldsymbol{x} \cdot \mathbb{C}^{N}$ with this norm is a finitedimensional Hilbert space.

2) We identify linear mappings (operators) on signal spaces with matrix multiplication from the right $\boldsymbol{x} \mapsto \boldsymbol{x} * A$. The adjoint operator is represented as $\boldsymbol{x} \mapsto \boldsymbol{x} * A^{\mathrm{H}}$, where $A^{\mathrm{H}}$ is the conjugate transpose $A^{\mathrm{H}}$ of $A$.

3) The discrete time translation operator $T_{k}$ is taken to correspond to the cyclic rotation

$$
T_{k}(x):=(x(j-k))_{j=0}^{N-1} \text { for } k \in \mathbb{Z} .
$$

The discrete modulation operator $M_{l}$ is given as

$$
M_{l}(\boldsymbol{x}):=\left(x(j) w^{j}\right)_{j=0}^{N-1} \quad \text { for } \quad l \in \mathbb{Z}
$$

where $w=e^{-2 \pi i l / N}$.

\section{B. Discrete Gabor Representation}

Let $a$ and $b$ be integers that divide $N$. For a signal $\boldsymbol{x}$, a discrete Gabor representation of $\boldsymbol{x}$ with respect to a Gabor triple $(\boldsymbol{g}, a, b)$ [22] is a series expansion of the form

$$
\boldsymbol{x}=\sum_{n=0}^{(N / a)-1} \sum_{m=0}^{(N / b)-1} c_{n, m} \boldsymbol{g}_{n, m}
$$

where $\boldsymbol{g}_{n, m}=M_{m b} T_{n a} \boldsymbol{g}$ is a Gabor elementary function [22], [23], [32], $\left\{\boldsymbol{g}_{n, m}\right\}_{n, m}$ is called a Gabor family [22], [27], and $\left\{c_{n, m}\right\}_{n, m}$ is a set of Gabor coefficients. We call $a$ and $b$ the lattice constants and $\tilde{a}=(N / a)$ and $\tilde{b}=(N / b)$ the dual lattice constants. The (discrete) Gabor operator $\mathcal{S}$ associated with $(\boldsymbol{g}, a, b)$ is defined by

$$
\mathcal{S} x=\sum_{n=0}^{(N / a)-1} \sum_{m=0}^{(N / b)-1}\left\langle\boldsymbol{x}, \boldsymbol{g}_{n, m}\right\rangle \boldsymbol{g}_{n, m} .
$$

Depending on the number of points in the time-frequency (TF) plane, we have the following situations.

- The case in which $(a, b)$ satisfies $a b=N$ is called critical sampling.

- The case in which $(a, b)$ satisfies $a b<N$ is called oversampling.

- The case in which $(a, b)$ satisfies $a b>N$ is called undersampling.

There is much in the literature concerning the first two cases. Many interesting results and algorithms have been obtained. We refer to [9], [11], [16], [17], [19], [20], [22], [23], [28]-[30] for detailed information.

However, we have not noticed much study on undersampled cases. We are motivated to fill this gap in this paper. We develop close connections between the undersampled and oversampled cases. Then, relevant algorithms are derived. 
The organization of this paper is as follows. In Section II, we introduce the generalized dual Gabor window (GDGW) function. We show that the GDGW plays a role similar to the dual Gabor window. It can be used to determine a set of Gabor coefficients. Then, we are able to obtain the best approximations of signals by linear combinations of vectors in the Gabor family. The main results are stated in Section III. We formulate relations between the undersampled DGT and the oversampled DGT. The undersampled GDGW (GTGW) is obtained by the dual (tight) Gabor window in the appropriate oversampled setting. There is a key lemma, which may be interesting by itself in linear algebra. All the technical proofs are presented in the Appendices. In Section IV, we propose relevant numerical algorithms and simulations. We give a brief conclusion in Section V.

\section{Generalized DuAl Gabor Window (GDGW)} [22].

In order to define the GDGW, we need more notation from

For a Gabor triple $(\boldsymbol{g}, a, b)$, we define the Gabor basic matrix $\operatorname{GAB}(\boldsymbol{g}, a, b)$ as the block matrix form

$$
\text { the } k \text { th row of } \operatorname{GAB}(\boldsymbol{g}, a, b)=M_{m b} T_{n a} \boldsymbol{g}
$$

where $k=(m \tilde{a}+n+1), m=0,1, \cdots, \tilde{b}-1, n=0,1, \cdots, \tilde{a}-$ 1 so that $k=1,2, \cdots, \tilde{N}(\tilde{N}=\tilde{a} \tilde{b})$. It is clear that $\operatorname{GAB}(\boldsymbol{g}, a, b)$ is an $\tilde{N}$-by- $N$ matrix. $\boldsymbol{g}$ and $T_{a} \boldsymbol{g}$ are the first and second rows of the Gabor basic matrix.

Immediately, we have the matrix representation of the Gabor operator $\mathcal{S}$ as

$$
\mathcal{S} x=\boldsymbol{x} * S, \text { for any signal } \boldsymbol{x} \in \mathbb{C}^{N}
$$

where $\boldsymbol{S}=[\mathrm{GAB}(\boldsymbol{g}, a, b)]^{\mathrm{H}} *[\mathrm{GAB}(\boldsymbol{g}, a, b)]$ is called the Gabor matrix, and $*$ denotes the matrix multiplication.

Clearly, $S$ is positive semi-definite [14], [26]. By wellknown results on frames (we refer to [7] and [13] for details on frames) and standard linear algebra, the following are equivalent.

1) A Gabor family $\left\{\boldsymbol{g}_{n, m}\right\}_{n=0,1, \cdots, \tilde{a}-1 ; m=0,1, \cdots, \tilde{b}-1}$ is a frame in $\mathbb{C}^{N}$.

2) The linear span of the family is the whole space $\mathbb{C}^{N}$.

3) $S$ and $S$ are invertible.

4) $S$ is positive definite.

If $\left\{\boldsymbol{g}_{n, m}\right\}_{n, m}$ is a frame, then $\tilde{\boldsymbol{g}}=S^{-1} \boldsymbol{g}$ and $\boldsymbol{g}_{\boldsymbol{t}}=S^{-(1 / 2)} \boldsymbol{g}$ are well defined. $\tilde{\boldsymbol{g}}$ and $\boldsymbol{g}_{\boldsymbol{t}}$ are called the dual Gabor (analysis) window and tight Gabor window functions. Then, the Gabor coefficients in (2) are obtained as $c_{n, m}=\left\langle\boldsymbol{x}, \tilde{\boldsymbol{g}}_{n, m}\right\rangle$ for $n=0,1, \cdots, \tilde{a}-1$ and $m=0,1, \cdots, \tilde{b}-1$. The authors in [9], [11], [16], [17], [19], [20], [22], [23], and [28]-[30] have derived interesting approaches to determining the dual Gabor window $\tilde{\boldsymbol{g}}$.

In the undersampled case, how can we represent or approximate a signal $\boldsymbol{x}$ in the form of (2)? Can we define $\tilde{\boldsymbol{g}}$ in the same way so that we can determine a set of coefficients $\left\{c_{n, m}=\right.$ $\left.\left\langle\boldsymbol{x}, \tilde{\boldsymbol{g}}_{n, m}\right\rangle\right\}_{n, m}$ ? The answer is no. In fact, $a b>N$ implies $\tilde{N}=$ $\left(\frac{N^{2}}{a b}\right)<N$. The rank of $S=[\mathrm{GAB}(\boldsymbol{g}, a, b)]^{\mathrm{H}} *[\mathrm{GAB}(\boldsymbol{g}, a, b)]$ is at most $\tilde{N}<N$. The $N$-by- $N$ matrix $S$ is not invertible, and $S^{-1}$ does not exist.

However, the pseudo-inverse matrix $\operatorname{pinv}(S)$ is uniquely defined [25], [26] [the definition of $\operatorname{pinv}(S)$ is given in (20)]. We define

$$
\tilde{\boldsymbol{g}}:=\boldsymbol{g} * \operatorname{pinv}(\boldsymbol{S})
$$

as the generalized dual Gabor window (GDGW). Since $S$ is positive semi-definite, $S^{1 / 2},(\operatorname{pinv}(S))^{1 / 2}$ and $\operatorname{pinv}\left(S^{1 / 2}\right)$ are well defined [14]. We define

$$
\boldsymbol{g}_{\boldsymbol{t}}:=\boldsymbol{g} *(\operatorname{pinv}(\boldsymbol{S}))^{1 / 2}=\boldsymbol{g} * \operatorname{pinv}\left(\boldsymbol{S}^{1 / 2}\right)
$$

as the generalized tight Gabor window (GTGW).

The following result has extended the result presented in [8, p. 971].

Proposition 1: For a Gabor triple $(\boldsymbol{g}, a, b)$, let $\boldsymbol{S}=$ $[\mathrm{GAB}(\boldsymbol{g}, a, b)]^{\mathrm{H}} *[\mathrm{GAB}(\boldsymbol{g}, a, b)]$. Then, both the actions of $S$ and $\operatorname{pinv}(S)$ commute with $M_{m b}$ and $T_{n a}$ for $m, n \in \mathbb{Z}$ in the sense that

$$
\left(M_{m b} T_{n a} \boldsymbol{x}\right) * S=M_{m b} T_{n a}(\boldsymbol{x} * S), \text { for any } \boldsymbol{x} \in \mathbb{C}^{N}
$$

and

$$
\begin{aligned}
& \left(M_{m b} T_{n a} \boldsymbol{x}\right) * \operatorname{pinv}(\boldsymbol{S}) \\
& \quad=M_{m b} T_{n a}(\boldsymbol{x} * \operatorname{pinv}(S)), \text { for any } \boldsymbol{x} \in \mathcal{L}_{(\boldsymbol{g}, a, b)}
\end{aligned}
$$

where $\mathcal{L}_{(\boldsymbol{g}, a, b)}$ is the linear subspace spanned by the Gabor family $\left\{\boldsymbol{g}_{n, m}: n=0,1, \cdots, \tilde{a}-1 ; m=0,1, \cdots, \widetilde{b}-1\right\}$ over $\mathbb{C}$.

In particular

$$
\widetilde{\boldsymbol{g}_{n, m}}=\widetilde{\boldsymbol{g}}_{n, m}
$$

where $\widetilde{\boldsymbol{g}_{n, m}}=\boldsymbol{g}_{n, m} * \operatorname{pinv}(\boldsymbol{S})=\left(M_{m b} T_{n a} \boldsymbol{g}\right) * \operatorname{pinv}(\boldsymbol{S})$, and $\tilde{\boldsymbol{g}}_{n, m}=M_{m b} T_{n a} \tilde{\boldsymbol{g}}$.

Moreover, $\tilde{\boldsymbol{g}}_{n, m} \in \mathcal{L}_{(\boldsymbol{g}, a, b)}$.

Proof: See Appendix A.

For a signal $x \in \mathbb{C}^{N}$, we say the unique $x_{\text {app }}$ is the best approximation of $\boldsymbol{x}$ from the Gabor space $\mathcal{L}_{(\boldsymbol{g}, a, b)}$ if $\boldsymbol{x}_{\mathrm{app}} \in \mathcal{L}_{(\boldsymbol{g}, a, b)}$, and

$$
\left\|\boldsymbol{x}-\boldsymbol{x}_{\text {app }}\right\|=\min _{\boldsymbol{y} \in \mathcal{L}_{(\boldsymbol{g}, a, b)}}\{\|\boldsymbol{x}-\boldsymbol{y}\|\} .
$$

If $\tilde{\boldsymbol{g}}$ is known, the following proposition shows how to obtain $\boldsymbol{x}_{\text {app}}$.

Proposition 2: The best approximation of $\boldsymbol{x}$ from $\mathcal{L}_{(\boldsymbol{g}, a, b)}$ can be represented as

$$
\boldsymbol{x}_{\mathrm{app}}=\sum_{n=0}^{\tilde{a}-1} \sum_{m=0}^{\tilde{b}-1} c_{n, m} \boldsymbol{g}_{n, m} \quad \text { for } \quad c_{n, m}=\left\langle\boldsymbol{x}, \tilde{\boldsymbol{g}}_{n, m}\right\rangle .
$$

In particular

$$
\boldsymbol{x}=\sum_{n=0}^{\tilde{a}-1} \sum_{m=0}^{\tilde{b}-1}\left\langle\boldsymbol{x}, \tilde{\boldsymbol{g}}_{n, m}\right\rangle \boldsymbol{g}_{n, m}, \quad \text { for } \quad \boldsymbol{x} \in \mathcal{L}_{(\boldsymbol{g}, a, b)} .
$$

Proof: See Appendix B.

Using the arguments of the proofs of Propositions 1 and 2, the following corollaries are easy to verify. 
Corollary 1: The action of $\operatorname{pinv}\left(S^{1 / 2}\right)$ commutes with both $M_{m b}$ and $T_{n a}$ for $m, n \in \mathbb{Z}$. Consequently, $\left(g_{t}\right)_{n, m}:=M_{m b} T_{n a} g_{t}=M_{m b} T_{n a}\left(\boldsymbol{g} * \operatorname{pinv}\left(\boldsymbol{S}^{1 / 2}\right)\right) \in \mathcal{L}_{(\boldsymbol{g}, a, b)}$ and $M_{m b} T_{n a} g_{t}=\left(M_{m b} T_{n a} \boldsymbol{g}\right) * \operatorname{pinv}\left(\boldsymbol{S}^{1 / 2}\right)$.

Corollary 2: For a signal $\boldsymbol{x}$, the best approximation from $\mathcal{L}_{(\boldsymbol{g}, a, b)}$ to $\boldsymbol{x}$ is determined as

$$
\boldsymbol{x}_{\mathrm{app}}=\sum_{n=0}^{\tilde{a}-1} \sum_{m=0}^{\tilde{b}-1} c_{n, m}\left(\boldsymbol{g}_{\boldsymbol{t}}\right)_{n, m} \quad \text { for } \quad c_{n, m}=\left\langle\boldsymbol{x},\left(\boldsymbol{g}_{\boldsymbol{t}}\right)_{n, m}\right\rangle .
$$

\section{UNDERSAMPLED GABOR TRANSFORM AND ITS} RELATION TO THE OVERSAMPLED GABOR TRANSFORM

In this section, we present the main results that reveal relations between undersampled and oversampled Gabor transforms. Then, we are able to compute the undersampled GDGW and GTGW. We need the following lemma, which is of interest by itself in linear algebra.

Lemma 1: Let $S_{1}$ and $S_{2}$ be positive semi-definite (Hermitian) matrices in $\mathbb{C}^{N \times N}$. Then, there exists a real polynomial $p(t)$ of degree $\leq 2 N$ such that

$$
\operatorname{pinv}\left(S_{1}\right)=p\left(S_{1}\right) \quad \text { and } \quad \operatorname{pinv}\left(S_{2}\right)=p\left(S_{2}\right)
$$

Moreover, if there is a signal $\boldsymbol{x} \in \mathbb{C}^{N}$ satisfying

$$
\boldsymbol{x} * \boldsymbol{S}_{1}^{k}=\boldsymbol{x} * \boldsymbol{S}_{2}^{k}, \quad \text { for } \quad k=1,2, \cdots, 2 N
$$

then

$$
\boldsymbol{x} * \operatorname{pinv}\left(S_{1}\right)=\boldsymbol{x} * \operatorname{pinv}\left(\boldsymbol{S}_{2}\right) .
$$

Proof: See Appendix C.

Theorem A is obtained in [22, Algorithm 2]. We reformulate it in order to keep this paper self-contained.

Theorem A [22]: Assume that $S=\left[\operatorname{GAB}\left(g_{1}, a, b\right)\right]^{\mathrm{H}} *$ $\left[\mathrm{GAB}\left(\boldsymbol{g}_{2}, a, b\right)\right]$; then, for a signal $\boldsymbol{x}=(x(j))_{j=0}^{N-1}, \boldsymbol{y}=$ $\boldsymbol{x} * \boldsymbol{S}:=(y(j))_{j=0}^{N-1}$ is given as

$$
\begin{aligned}
y(j) & =\tilde{b} \sum_{p=0}^{b-1} x(j+p \tilde{b})\left\{\sum_{n=0}^{\tilde{a}-1} \overline{T_{n a} g_{1}\left(p \tilde{b}+j_{0}\right)} T_{n a} g_{2}\left(j_{0}\right)\right\} \\
& =\tilde{b} \sum_{p=0}^{b-1} x(j+p \tilde{b})\left\{\sum_{n=0}^{\tilde{a}-1} \overline{T_{n a} g_{1}(p \tilde{b}+j)} T_{n a} g_{2}(j)\right\}
\end{aligned}
$$

where $j=j_{0}+r a$ with $j_{0}=0,1, \cdots, a-1$ and $r=$ $0,1, \cdots, \tilde{a}-1$.

Note: Theorem A holds for all Gabor situations, whether oversampled, critically sampled, or undersampled.

Theorem 1: Let $S_{1}$ and $S_{2}$ be Gabor matrices corresponding to Gabor triples $(\boldsymbol{g}, a, b)$ and $(\boldsymbol{g}, \tilde{b}, \tilde{a})$, respectively. Then

$$
\boldsymbol{g} * \boldsymbol{S}_{1}^{k}=r^{k} \boldsymbol{g} * \boldsymbol{S}_{2}^{k} \quad \text { for } \quad k=1,2, \cdots
$$

where $r=\left(\frac{N}{a \cdot b}\right)$ is the redundancy of the TF lattice given by $(a, b)$.

Proof: See Appendix D.
Theorem 2-Undersampled Generalized Dual Gabor: Let $(\boldsymbol{g}, a, b)$ be a Gabor triple with $a b>N, \tilde{\boldsymbol{g}}_{1}$, and let $\tilde{\boldsymbol{g}}_{2}$ be the generalized dual Gabor windows associated with $(g, a, b)$ and $(\boldsymbol{g}, \tilde{b}, \tilde{a})$, respectively; then, $\tilde{\boldsymbol{g}}_{1}$ coincides with $\tilde{\boldsymbol{g}}_{2}$ up to the constant factor $a b / N$

$$
\tilde{\boldsymbol{g}}_{1}=\frac{a b}{N} \tilde{\boldsymbol{g}}_{2}
$$

If $(\boldsymbol{g}, \tilde{b}, \tilde{a})$ generates a frame, then $\tilde{\boldsymbol{g}}_{2}$ is the dual Gabor window, and (16) holds.

Proof: It follows from Lemma 1 and Theorem 1 that

$$
\begin{aligned}
\tilde{\boldsymbol{g}}_{1} & =\boldsymbol{g} * \operatorname{pinv}\left(\boldsymbol{S}_{1}\right)=\frac{a \cdot b}{N} \boldsymbol{g} * \operatorname{pinv}\left(\boldsymbol{S}_{2}\right) \\
& =\boldsymbol{g} * \operatorname{pinv}\left(\frac{N}{a \cdot b} * \boldsymbol{S}_{2}\right)=\frac{a \cdot b}{N} \boldsymbol{g} * \operatorname{pinv}\left(\boldsymbol{S}_{2}\right) \\
& =\frac{a \cdot b}{N} \tilde{\boldsymbol{g}}_{2} .
\end{aligned}
$$

Similarly, we derive a relation between the undersampled GTGW and oversampled tight Gabor window based on the binomial expansion of the inverse square root.

Theorem 3-Undersampled Generalized Tight Gabor: Let $\boldsymbol{g}_{\boldsymbol{t}}^{(1)}$ and $\boldsymbol{g}_{\boldsymbol{t}}^{(2)}$ be the generalized tight Gabor windows corresponding to $(\boldsymbol{g}, a, b)$ and $(\boldsymbol{g}, \tilde{b}, \tilde{a})$, respectively. Then

$$
\boldsymbol{g}_{\boldsymbol{t}}^{(1)}=\left(\frac{a \cdot b}{N}\right)^{1 / 2} \boldsymbol{g}_{\boldsymbol{t}}^{(2)}
$$

If $(\boldsymbol{g}, \tilde{b}, \tilde{a})$ generates a Gabor frame, $\boldsymbol{g}_{\boldsymbol{t}}^{(2)}$ is the tight Gabor window, and (17) holds.

\section{NumERICAL AlgORITHMS AND SimUlations}

Algorithm 1: For a Gabor triple $(g, a, b)$ with $a \cdot b>N$ and a signal $\boldsymbol{x} \in \mathbb{C}^{N}$, suppose that $(\boldsymbol{g}, \tilde{b}, \tilde{a})$ generates a Gabor frame; then, the GDGW and the best approximation $\boldsymbol{x}_{\mathrm{app}}$ from the Gabor space $\mathcal{L}_{(\boldsymbol{g}, a, b)}$ are determined by the following.

$G D G W$ and $G T G W$ : The rich structures of oversampled Gabor matrices allow us to determine the dual Gabor window $\tilde{\boldsymbol{g}}_{0}$ and tight Gabor window $\boldsymbol{g}_{\boldsymbol{t}_{o}}$ corresponding to $(\boldsymbol{g}, \tilde{b}, \tilde{a})$ [22], [23]. Applying Theorem 2

$$
\tilde{\boldsymbol{g}}=\frac{a b}{N} \tilde{\boldsymbol{g}}_{0}
$$

and

$$
g_{t}=\sqrt{\frac{a b}{N}} g_{t_{0}}
$$

$x_{\mathrm{app}}$ by $G D G W$ : We calculate the Gabor coefficients as

$$
c_{n, m}=\left\langle\boldsymbol{x}, \tilde{\boldsymbol{g}}_{n, m}\right\rangle=\sum_{k=0}^{N-1} x(k) \overline{\tilde{\boldsymbol{g}}_{n, m}(k)}
$$


which can be done efficiently using the short time Fourier transform (STFT).

Then, $x_{\text {app }}$ is obtained as

$$
\boldsymbol{x}_{\mathrm{app}}=\sum_{n=0}^{\tilde{a}-1} \sum_{m=0}^{\tilde{b}-1} c_{n, m} \boldsymbol{g}_{n, m} \text {. }
$$

In particular, if $\boldsymbol{x} \in \mathcal{L}_{(\boldsymbol{g}, a, b)}$

$$
\boldsymbol{x}=\boldsymbol{x}_{\mathrm{app}}=\sum_{n=0}^{\tilde{a}-1} \sum_{m=0}^{\tilde{b}-1} c_{n, m} \boldsymbol{g}_{n, m}
$$

$x_{\text {app }}$ by $G T G W$ :

$$
\boldsymbol{x}_{\mathrm{app}}=\sum_{n=0}^{\tilde{a}-1} \sum_{m=0}^{\tilde{b}-1}\left\langle\boldsymbol{x},\left(\boldsymbol{g}_{\boldsymbol{t}}\right)_{n, m}\right\rangle\left(\boldsymbol{g}_{\boldsymbol{t}}\right)_{n, m} .
$$

For $\boldsymbol{x} \in \mathcal{L}_{(\boldsymbol{g}, a, b)}$

$$
\boldsymbol{x}=\boldsymbol{x}_{\mathrm{app}}=\sum_{n=0}^{\tilde{a}-1} \sum_{m=0}^{\tilde{b}-1}\left\langle\boldsymbol{x},\left(\boldsymbol{g}_{\boldsymbol{t}}\right)_{n, m}\right\rangle\left(\boldsymbol{g}_{\boldsymbol{t}}\right)_{n, m}
$$

The algorithm is easily derived from the results of Sections II and III.

By Algorithm 1, the computations of undersampled DGT are made possible. For an undersampled Gabor triple $(\boldsymbol{g}, a, b)$ with $a b>N$, we need to consider the corresponding oversampled case with $(\boldsymbol{g}, \tilde{b}, \tilde{a})$. By employing algorithms in [22] and [23], we determine the dual (tight) Gabor window function $\tilde{\boldsymbol{g}}_{o}\left(\boldsymbol{g}_{\boldsymbol{t}_{o}}\right)$ associated with $(\boldsymbol{g}, \tilde{b}, \tilde{a})$. By Algorithm 1, the GDGW $\tilde{\boldsymbol{g}}$ associated with $(\boldsymbol{g}, a, b)$ is given by $\tilde{\boldsymbol{g}}=(a b / N) \tilde{\boldsymbol{g}}_{o}$, whereas the GTGW $\boldsymbol{g}_{\boldsymbol{t}}=\sqrt{(a b / N)} \boldsymbol{g}_{\boldsymbol{t}}$. Then, for a signal $\boldsymbol{x}$, the best approximation $\boldsymbol{x}_{\mathrm{app}}$ is determined by using the GDGW $\tilde{\boldsymbol{g}}$ (GTGW $\boldsymbol{g}_{\boldsymbol{t}}$ ).

In the following, we present some numerical simulations related to the computations of undersampled DGT.

Fig. 1 shows a Gabor window of signal length $N=600$ with lattice constants $a=40$ and $b=25$. Since $40 \times 25=$ $1000>600$, it is undersampling. The undersampled GDGW and GTGW are illustrated. Using GDGW and GTGW, we are able to obtain the best approximations $\boldsymbol{x}_{\mathrm{app}}^{(d)}$ and $\boldsymbol{x}_{\mathrm{app}}^{(t)}$ from the Gabor space to a signal $\boldsymbol{x}$. Fig. 2 shows the original signal $\boldsymbol{x}$ and the best approximations $\boldsymbol{x}_{\mathrm{app}}^{(d)}$ and $\boldsymbol{x}_{\mathrm{app}}^{(t)}$. The relative errors $\left\|\boldsymbol{x}-\boldsymbol{x}_{\mathrm{app}}^{(d)}\right\| /\|\boldsymbol{x}\|$ and $\left\|\boldsymbol{x}-\boldsymbol{x}_{\mathrm{app}}^{(t)}\right\| /\|\boldsymbol{x}\|$ are about $1.0261 \mathrm{e}-13$ and $1.1502 \mathrm{e}-14$, respectively.

Fig. 3 shows the Gabor window and the GDGW and GTGW with large signal length $N=2520$. The lattice constants $a=70$ and $b=56$. Clearly, $70 \times 56=3920>2520$; therefore, it is undersampling. Fig. 4 illustrates an original Chirp signal and the best approximations $\boldsymbol{x}_{\mathrm{app}}^{(d)}$ and $\boldsymbol{x}_{\mathrm{app}}^{(t)}$ from the Gabor space to the Chirp. $\boldsymbol{x}_{\mathrm{app}}^{(d)}$ and $\boldsymbol{x}_{\mathrm{app}}^{(t)}$ are computed with the dual
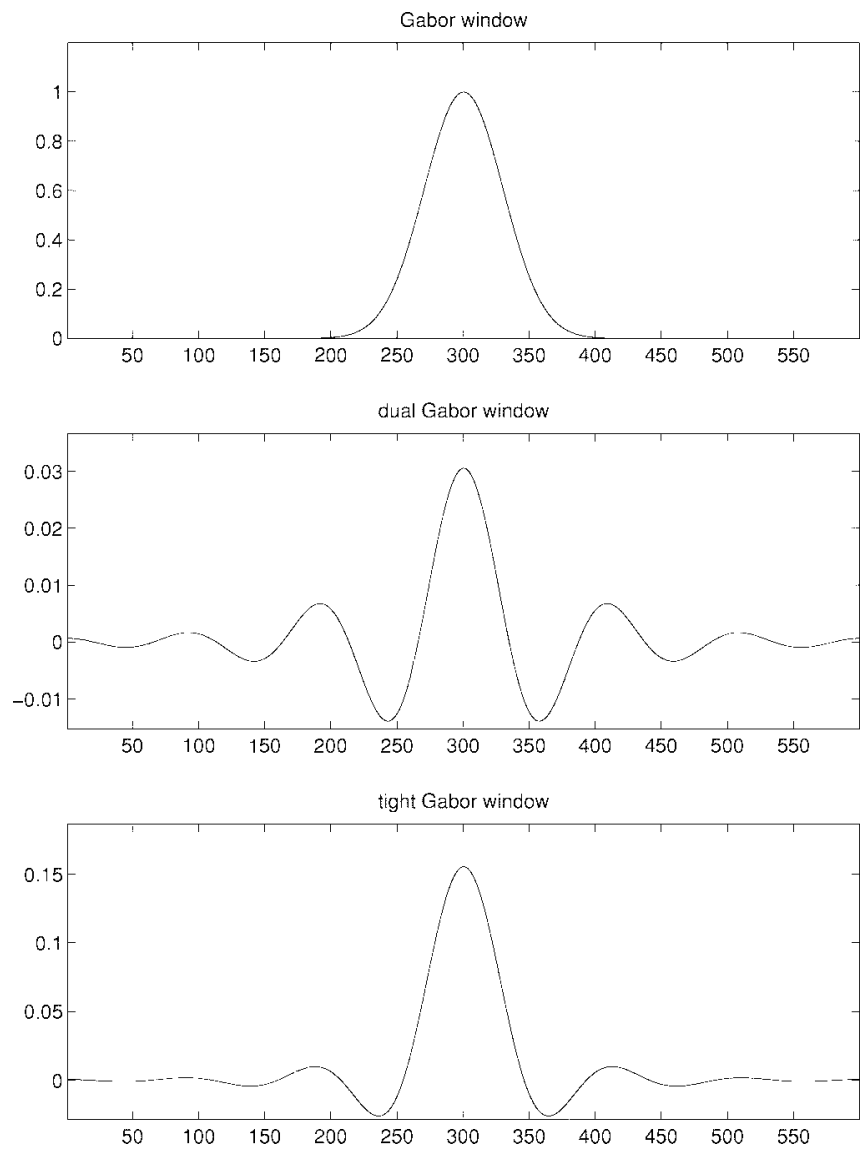

Fig. 1. Gabor window and the associated GDGW and GTGW. The signal length is 600 and the lattice constants $a=40, b=25$.

Gabor window and tight Gabor window, respectively. They well approximate the original Chirp.

\section{CONCLUSION}

We have developed relations between undersampled and oversampled Gabor transforms. The introduced GDGW function is similar to the dual Gabor (analysis) window. It is used to determine a set of Gabor coefficients. We proved that the undersampled GDGW is the same as the oversampled dual Gabor window with respect to dual lattice constants up to a constant factor. Similar results were derived for the GTGW. This makes it possible to compute the undersampled DGT.

\section{APPENDIX A \\ PROOF OF PROPOSITION 1}

Proof: Following the arguments used in [10, Th. 2], we can easily check that (4) holds.

Since the matrix $\boldsymbol{S}=[\mathrm{GAB}(\boldsymbol{g}, a, b)]^{\mathrm{H}} *[\mathrm{GAB}(\boldsymbol{g}, a, b)]$ for any signal $\boldsymbol{x} \in \mathcal{L}_{(\boldsymbol{g}, a, b)}$, we see that $\boldsymbol{x}$ is in the row space of the Gabor matrix $S$. Therefore (cf. [25, pp. 853-854] and [26, pp. 137-140])

$$
\begin{aligned}
\{\boldsymbol{x} * \operatorname{pinv}(\boldsymbol{S})\} * \boldsymbol{S} & =\boldsymbol{x} *\{\operatorname{pinv}(\boldsymbol{S}) * \boldsymbol{S}\} \\
& =\boldsymbol{x} \text { for } \boldsymbol{x} \in \mathcal{L}_{(\boldsymbol{g}, a, b)}
\end{aligned}
$$



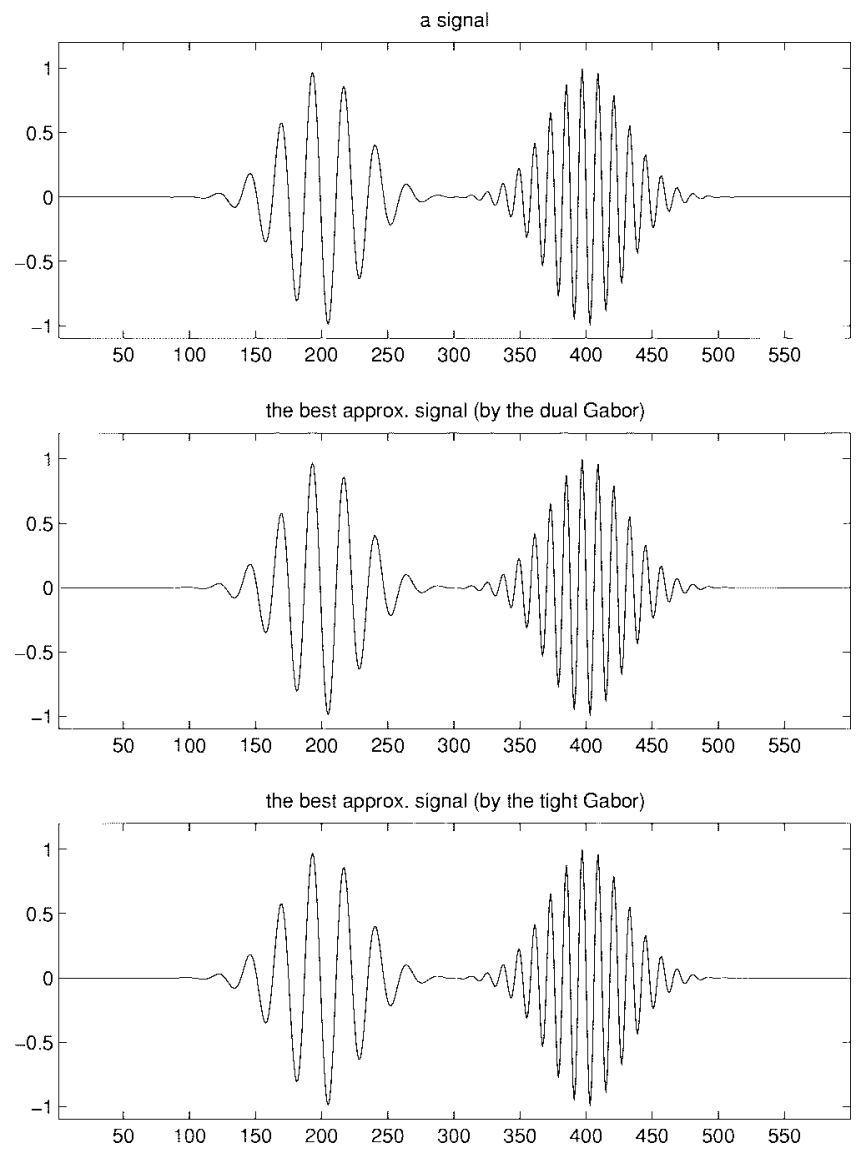

Fig. 2. Original signal and the best approximate signals from the Gabor space. The signal length is 600 .

Since $S$ and pinv $(S)$ are Hermitian, we deduce that [26, p. 145]

$$
\begin{aligned}
\{\boldsymbol{x} * S\} * \operatorname{pinv}(\boldsymbol{S}) & =\{\boldsymbol{x} * \operatorname{pinv}(\boldsymbol{S})\} * S \\
& =\boldsymbol{x} \text { for } \boldsymbol{x} \in \mathcal{L}_{(\boldsymbol{g}, a, b)} .
\end{aligned}
$$

Using (4) and (19), we derive that

$$
\begin{aligned}
& M_{m b} T_{n a}(\boldsymbol{x} * \operatorname{pinv}(S)) \\
& \quad=M_{m b} T_{n a}(\boldsymbol{x} * \operatorname{pinv}(S)) *\{\boldsymbol{S} * \operatorname{pinv}(\boldsymbol{S})\} \\
& \quad=M_{m b} T_{n a}\{\boldsymbol{x} * \operatorname{pinv}(\boldsymbol{S}) * \boldsymbol{S}\} * \operatorname{pinv}(\boldsymbol{S}) \\
& \quad=\left(M_{m b} T_{n a} \boldsymbol{x}\right) * \operatorname{pinv}(\boldsymbol{S}) .
\end{aligned}
$$

Therefore, (5) holds. Taking $\boldsymbol{x}=\boldsymbol{g}$ in (5), we derive (6) easily.

Since $\boldsymbol{S}$ is positive semi-definite

$$
\begin{aligned}
\widetilde{\boldsymbol{g}}_{n, m}= & \widetilde{\boldsymbol{g}_{n, m}}=\boldsymbol{g}_{n, m} * \operatorname{pinv}(\boldsymbol{S}) \\
= & \boldsymbol{g}_{n, m} * \operatorname{pinv}(\boldsymbol{S}) * \boldsymbol{S} * \operatorname{pinv}(\boldsymbol{S}) \\
= & \boldsymbol{g}_{n, m} * \operatorname{pinv}\left(\boldsymbol{S}^{2}\right) * \boldsymbol{S} \\
= & \boldsymbol{g}_{n, m} * \operatorname{pinv}\left(\boldsymbol{S}^{2}\right) *[\mathrm{GAB}(\boldsymbol{g}, a, b)]^{\mathrm{H}} \\
& *[\mathrm{GAB}(\boldsymbol{g}, a, b)] .
\end{aligned}
$$

By the construction of Gabor basic matrix $\operatorname{GAB}(\boldsymbol{g}, a, b)$, we deduce that $\tilde{\boldsymbol{g}}_{n, m} \in \mathcal{L}_{(\boldsymbol{g}, a, b)}$.
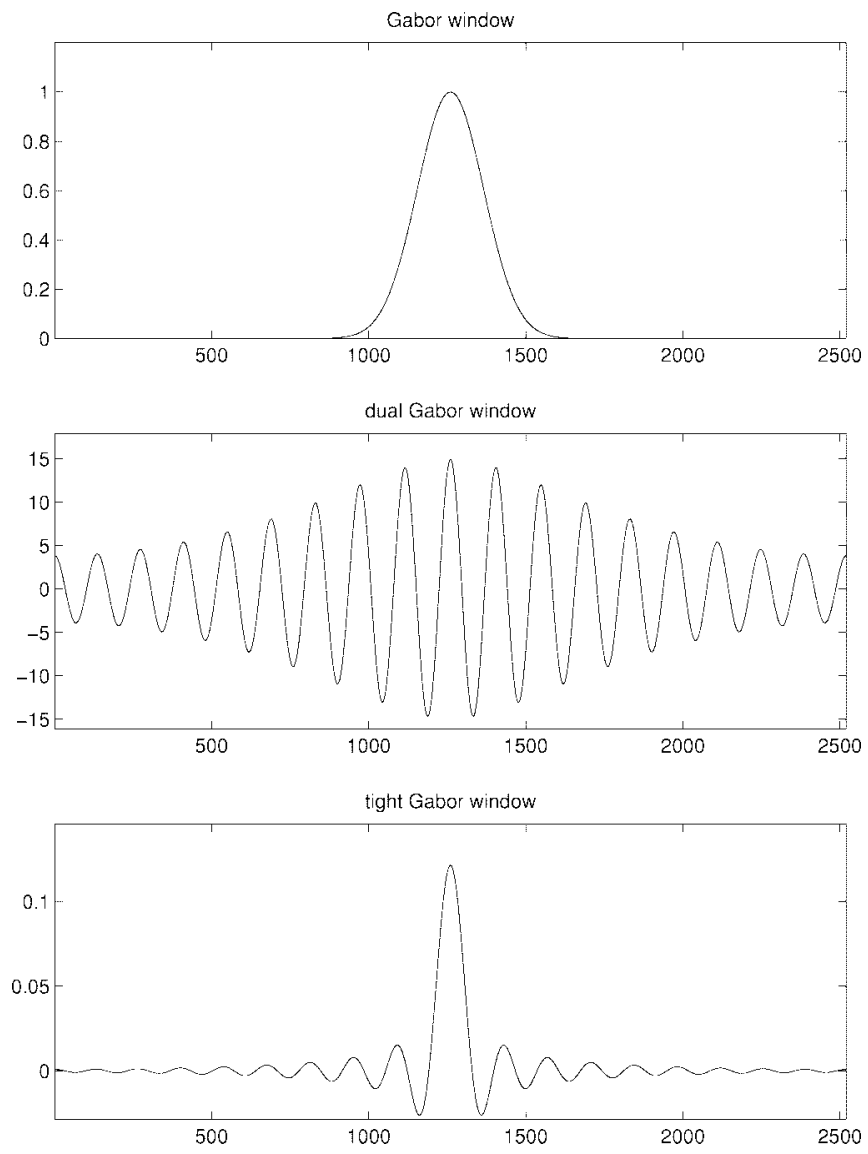

Fig. 3. Gabor window and the associated GDGW and GTGW. The signal length is 2520 , and the lattice constants $a=70, b=56$.

\section{APPENDIX B}

PROOF OF PROPOSITION 2

Proof: We show the special case (8) first. For $\boldsymbol{x} \in$ $\mathcal{L}_{(\boldsymbol{g}, a, b)}$, using (6)

$$
\begin{aligned}
\sum_{n=0}^{\tilde{a}-1} \sum_{m=0}^{\tilde{b}-1}\left\langle\boldsymbol{x}, \tilde{\boldsymbol{g}}_{n, m}\right\rangle \boldsymbol{g}_{n, m} \\
\quad=\sum_{n=0}^{\tilde{a}-1} \sum_{m=0}^{\tilde{b}-1}\left\langle\boldsymbol{x}, \boldsymbol{g}_{n, m} * \operatorname{pinv}(\boldsymbol{S})\right\rangle \boldsymbol{g}_{n, m} \\
\quad=\sum_{n=0}^{\tilde{a}-1} \sum_{m=0}^{\tilde{b}-1}\left\langle\boldsymbol{x} * \operatorname{pinv}\left(\boldsymbol{S}^{\mathrm{H}}\right), \boldsymbol{g}_{n, m}\right\rangle \boldsymbol{g}_{n, m} \\
\quad=\sum_{n=0}^{\tilde{a}-1} \sum_{m=0}^{\tilde{b}-1}\left\langle\boldsymbol{x} * \operatorname{pinv}(\boldsymbol{S}), \boldsymbol{g}_{n, m}\right\rangle \boldsymbol{g}_{n, m} \\
=\{\boldsymbol{x} * \operatorname{pinv}(\boldsymbol{S})\} * \boldsymbol{S} .
\end{aligned}
$$

Using (18), we conclude that

$$
\boldsymbol{x}=\{\boldsymbol{x} * \operatorname{pinv}(\boldsymbol{S})\} * \boldsymbol{S}=\sum_{n=0}^{\tilde{a}-1} \sum_{m=0}^{\tilde{b}-1}\left\langle\boldsymbol{x}, \tilde{\boldsymbol{g}}_{n, m}\right\rangle \boldsymbol{g}_{n, m} .
$$

In order to verify the general case (7), let us look at $\boldsymbol{x}_{\mathrm{app}}$ in another way. Suppose that the mapping $P: \mathbb{C}^{N} \vdash \mathcal{L}_{(\boldsymbol{g}, a, b)}$ is the orthogonal projection. Then $x_{\mathrm{app}}=P(\boldsymbol{x})$. 

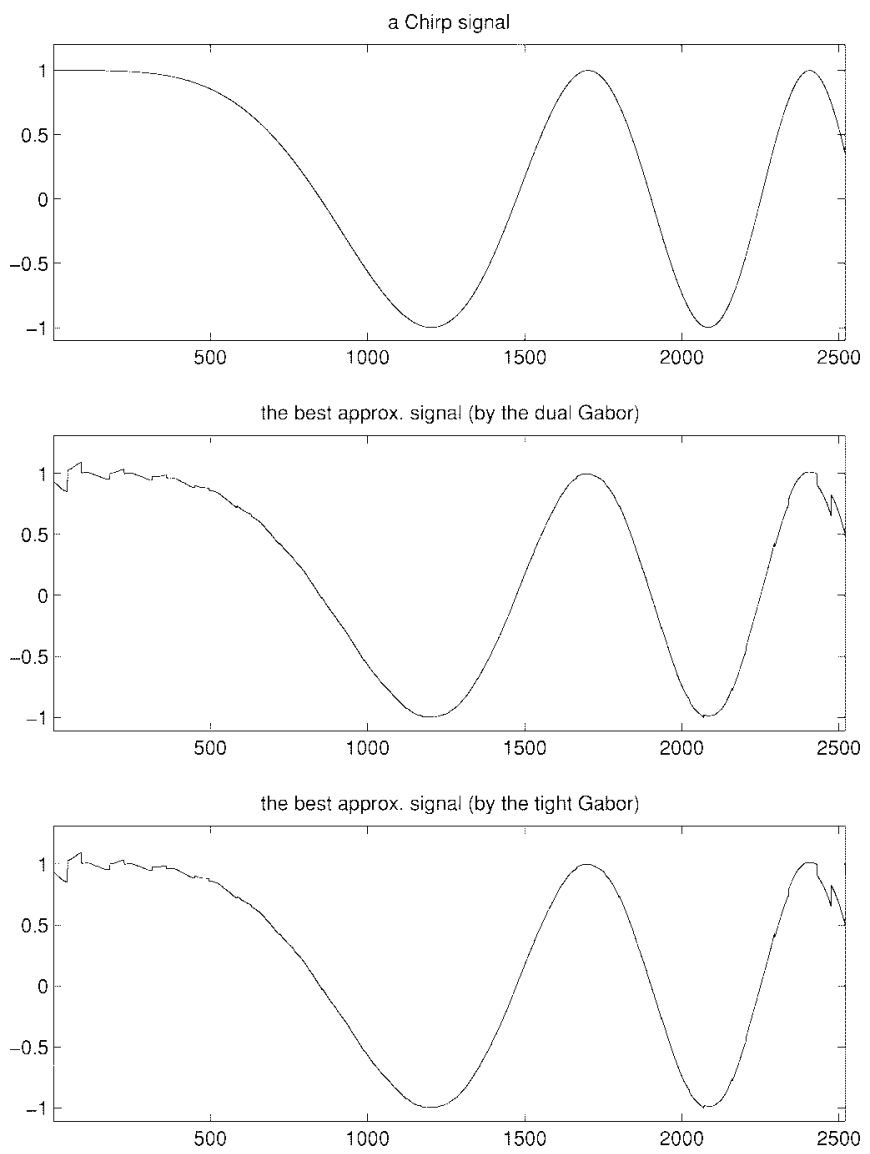

Fig. 4. Original Chirp signal and the best approximate signals from the Gabor space. The signal length is 2520 .

Since $P$ is the orthogonal projection and $\tilde{\boldsymbol{g}}_{n, m} \in \mathcal{L}_{(\boldsymbol{g}, a, b)}$ by Proposition 1

$$
\begin{aligned}
P(\boldsymbol{x}) & \in \mathcal{L}_{(\boldsymbol{g}, a, b)}, \quad \text { and } \\
\left\langle P(\boldsymbol{x}), \tilde{\boldsymbol{g}}_{n, m}\right\rangle & =\left\langle\boldsymbol{x}, P\left(\tilde{\boldsymbol{g}}_{n, m}\right)\right\rangle=\left\langle\boldsymbol{x}, \tilde{\boldsymbol{g}}_{n, m}\right\rangle .
\end{aligned}
$$

It follows by using (8) that

$$
\begin{aligned}
\boldsymbol{x}_{\mathrm{app}} & =P(\boldsymbol{x})=\sum_{n=0}^{\tilde{a}-1} \sum_{m=0}^{\tilde{b}-1}\left\langle P(\boldsymbol{x}), \tilde{\boldsymbol{g}}_{n, m}\right\rangle \boldsymbol{g}_{n, m} \\
& =\sum_{n=0}^{\tilde{a}-1} \sum_{m=0}^{\tilde{b}-1}\left\langle\boldsymbol{x}, \tilde{\boldsymbol{g}}_{n, m}\right\rangle \boldsymbol{g}_{n, m} .
\end{aligned}
$$

APPENDIX C

PROOF OF LEMMA 1

Proof: Since both $\boldsymbol{S}_{1}$ and $\boldsymbol{S}_{2}$ are positive semi-definite Hermitian matrices, there exist diagonalizing unitary matrices $U_{1}$ and $U_{2}$ (see [26]) and diagonal matrices $D_{1}$ and $D_{2}$ with non-negative diagonal entries such that

$$
\boldsymbol{S}_{j}=U_{j}^{\mathrm{H}} * D_{j} * U_{j} \quad \text { for } \quad j=1,2 .
$$

Then, we have, by [26, p. 449] and [25]

$$
\operatorname{pinv}\left(\boldsymbol{S}_{j}\right)=U_{j}^{\mathrm{H}} * \operatorname{pinv}\left(D_{j}\right) * U_{j} \quad \text { for } \quad j=1,2 \text {. }
$$

Write

$$
D_{j}=\left(\begin{array}{cccc}
\lambda_{1}^{(j)} & & & \\
& \lambda_{2}^{(j)} & & \\
& & \ddots & \\
& & & \lambda_{N}^{(j)}
\end{array}\right) \text { for } j=1,2
$$

where $\lambda_{l}^{(j)} \geq 0$ for $l=1,2, \cdots, N$, and $j=1,2$. Assume that $\left\{\mu_{k}\right\}_{k=1}^{K}$ for some $1<K \leq 2 N$ are all the different numbers from $\left\{\lambda_{l}^{(j)}\right\}_{1 \leq l \leq N ; j=1,2}$. Then, there exists a real interpolation polynomial [15] $p(t)$ such that for $k=1,2, \cdots, K$

$$
p\left(\mu_{k}\right)= \begin{cases}\frac{1}{\mu_{k}} & \text { if } \mu_{k} \neq 0 \\ 0 & \text { if } \mu_{k}=0 .\end{cases}
$$

It follows immediately that for $l=1,2, \cdots, N$ and $j=1,2$

$$
p\left(\lambda_{l}^{(j)}\right)= \begin{cases}\frac{1}{\lambda_{l}^{(j)}} & \text { if } \lambda_{l}^{(j)} \neq 0 \\ 0 & \text { if } \lambda_{l}^{(j)}=0 .\end{cases}
$$

This implies that [25], [26] for $j=1,2$

$$
\operatorname{pinv}\left(D_{j}\right)=\left(\begin{array}{llll}
p\left(\lambda_{1}^{(j)}\right) & & & \\
& p\left(\lambda_{2}^{(j)}\right) & & \\
& & \ddots & \\
& & & p\left(\lambda_{N}^{(j)}\right)
\end{array}\right)=p\left(D_{j}\right)
$$

Thus

$$
\begin{aligned}
p\left(S_{1}\right) & =p\left(U_{1}^{\mathrm{H}} * D_{1} * U_{1}\right)=U_{1}^{\mathrm{H}} * p\left(D_{1}\right) * U_{1} \\
& =U_{1}^{\mathrm{H}} * p\left(D_{1}\right) * U_{1}=U_{1}^{\mathrm{H}} * \operatorname{pinv}\left(D_{1}\right) * U_{1} \\
& =\operatorname{pinv}\left(\boldsymbol{S}_{1}\right) .
\end{aligned}
$$

Hence, $\operatorname{pinv}\left(\boldsymbol{S}_{1}\right)=p\left(\boldsymbol{S}_{1}\right)$. Similarly, $\operatorname{pinv}\left(\boldsymbol{S}_{2}\right)=p\left(\boldsymbol{S}_{2}\right)$. This completes the proof of (10).

From (10) and (11), (12) is easy to derive. In fact

$$
\begin{aligned}
\boldsymbol{x} * \operatorname{pinv}\left(\boldsymbol{S}_{1}\right) & =\boldsymbol{x} * p\left(\boldsymbol{S}_{1}\right)=\boldsymbol{x} * p\left(\boldsymbol{S}_{2}\right) \\
& =\boldsymbol{x} * \operatorname{pinv}\left(\boldsymbol{S}_{2}\right) .
\end{aligned}
$$

\section{APPENDIX D}

\section{PROOF OF THEOREM 1}

Proof:

Step One: Let

$$
\boldsymbol{y}=\boldsymbol{g} * \boldsymbol{S}_{1} \quad \text { and } \quad \boldsymbol{z}=\boldsymbol{g} * \boldsymbol{S}_{2}
$$

and write $\boldsymbol{g}=(g(j))_{j=0}^{N-1}, \boldsymbol{y}=(y(j))_{j=0}^{N-1}$, and $\boldsymbol{z}=$ $(z(j))_{j=0}^{N-1}$ 
Using Theorem A and the $N$ periodicity of signals, for any $j=0,1, \cdots, N-1$, we have

$$
\begin{aligned}
y(j) & =\tilde{b} \sum_{p=0}^{b-1} g(j+p \tilde{b})\left\{\sum_{n=0}^{\tilde{a}-1} \overline{T_{n a} g(p \tilde{b}+j)} T_{n a} g(j)\right\} \\
& =\tilde{b} \sum_{p=0}^{b-1} \sum_{n=0}^{\tilde{a}-1}\{g(j+p \tilde{b}) \overline{g(p \tilde{b}+j+n a)} g(j+n a)\} \\
& =\tilde{b} \sum_{n=0}^{\tilde{a}-1} g(j+n a)\left\{\sum_{p=0}^{b-1} \overline{g(p \tilde{b}+n a+j)} g(j+p \tilde{b})\right\} \\
& =\tilde{b} \sum_{n=0}^{\tilde{a}-1} g(j+n a)\left\{\sum_{p=0}^{b-1} \overline{T_{p \tilde{b}} g(n a+j)} T_{p \tilde{b}} g(j)\right\} \\
& =\frac{\tilde{b}}{a}\left\{a \sum_{n=0}^{\tilde{a}-1} g(j+n a)\left\{\sum_{p=0}^{b-1} \overline{T_{p \tilde{b}} g(n a+j)} T_{p \tilde{b}} g(j)\right\}\right\} \\
& =\frac{N}{a \cdot b} z(j)=r \cdot z(j) .
\end{aligned}
$$

Thus, $\boldsymbol{g} * \boldsymbol{S}_{1}=r \boldsymbol{g} * \boldsymbol{S}_{2}$.

Step Two: Inductively, assume that for some $k \in \mathbb{N}$, we have

$$
\boldsymbol{y}:=\boldsymbol{g} * \boldsymbol{S}_{1}^{k}=r^{k} \boldsymbol{g} * S^{k} .
$$

We need to verify that

$$
\boldsymbol{g} * \boldsymbol{S}_{1}^{k+1}=r^{k+1} \boldsymbol{g} * \boldsymbol{S}^{k+1} .
$$

Write $\boldsymbol{z}=\boldsymbol{g} * \boldsymbol{S}_{2}^{k}, \boldsymbol{u}=\boldsymbol{g} * \boldsymbol{S}_{1}^{k+1}=\boldsymbol{y} * \boldsymbol{S}_{1}$, and $\boldsymbol{v}=\boldsymbol{g} * \boldsymbol{S}_{2}^{k+1}=$ $z * S_{2}$; then, for any $j=0,1, \cdots, N-1$

$$
\begin{aligned}
\left(\boldsymbol{g} * \boldsymbol{S}_{1}^{k+1}\right)(j) & u(j)=\tilde{b} \sum_{p=0}^{b-1} y(j+p \tilde{b})\left\{\sum_{n=0}^{\tilde{a}-1} \overline{T_{n a} g(p \tilde{b}+j)} T_{n a} g(j)\right\} \\
= & \tilde{b} r^{k} \sum_{p=0}^{b-1} \sum_{n=0}^{\tilde{a}-1}\{z(j+p \tilde{b}) \overline{g(p \tilde{b}+n a+j)} g(j+n a)\} \\
= & \tilde{b} r^{k} \sum_{n=0}^{\tilde{a}-1} g(j+n a)\left\{\sum_{p=0}^{b-1} \overline{g(p \tilde{b}+n a+j)} z(j+p \tilde{b})\right\} \\
= & \frac{\tilde{b}}{a} r^{k}\left\{a \sum _ { n = 0 } ^ { \tilde { a } - 1 } g ( j + n a ) \left\{\sum_{p=0}^{b-1} \overline{T_{p \tilde{b}} g(n a+j)}\right.\right. \\
& \left.\left.\cdot T_{p \tilde{b}} z(j)\right\}\right\} \\
= & r^{k+1}(\boldsymbol{g} * H)(j)
\end{aligned}
$$

where

$$
H=[\operatorname{GAB}(\boldsymbol{g}, \tilde{b}, \tilde{a})]^{\mathrm{H}} *[\operatorname{GAB}(z, \tilde{b}, \tilde{a})] .
$$

The last equation is derived from Theorem A.
Since

$$
\begin{aligned}
\boldsymbol{g} * H & =\sum_{n=0}^{b-1} \sum_{m=0}^{a-1}\left\langle\boldsymbol{g}, M_{m \tilde{a}} T_{n \tilde{b}} \boldsymbol{g}\right\rangle M_{m \tilde{\boldsymbol{a}}} T_{n \tilde{b}} \boldsymbol{z} \\
& =\sum_{n=0}^{b-1} \sum_{m=0}^{a-1}\left\langle\boldsymbol{g}, M_{m \tilde{a}} T_{n \tilde{b}} \boldsymbol{g}\right\rangle M_{m \tilde{\boldsymbol{a}}} T_{n \tilde{b}}\left(\boldsymbol{g} * \boldsymbol{S}_{2}^{k}\right) \\
& =\sum_{n=0}^{b-1} \sum_{m=0}^{a-1}\left\langle\boldsymbol{g}, M_{m \tilde{\boldsymbol{a}}} T_{n \tilde{b}} \boldsymbol{g}\right\rangle\left\{M_{m \tilde{\boldsymbol{a}}} T_{n \tilde{b}} \boldsymbol{g}\right\} * \boldsymbol{S}_{2}^{k} \\
& =\left\{\sum_{n=0}^{b-1} \sum_{m=0}^{a-1}\left\langle\boldsymbol{g}, M_{m \tilde{a}} T_{n \tilde{b}} \boldsymbol{g}\right\rangle M_{m \tilde{\boldsymbol{a}}} T_{n \tilde{b}} \boldsymbol{g}\right\} * \boldsymbol{S}_{2}^{k} \\
& =\left\{\boldsymbol{g} * \boldsymbol{S}_{2}\right\} * \boldsymbol{S}_{2}^{k}=\boldsymbol{g} * \boldsymbol{S}_{2}^{k+1}
\end{aligned}
$$

where the third equation is derived by using (4), we deduce that

$$
\begin{gathered}
u(j)=r^{k+1}(\boldsymbol{g} * H)(j)=r^{k+1}\left(\boldsymbol{g} * \boldsymbol{S}_{2}^{k+1}\right)(j)=v(j) \\
\text { for } j=0,1, \cdots, N-1 .
\end{gathered}
$$

Therefore

$$
\boldsymbol{g} * \boldsymbol{S}_{1}^{k+1}=r^{k+1} \boldsymbol{g} * \boldsymbol{S}_{2}^{k+1} .
$$

\section{ACKNOWLEDGMENT}

The author would like to thank Prof. H. G. Feichtinger for fruitful discussions on the subject. Special thanks go to J. W. Dunion for carefully reading the whole manuscript and making helpful comments.

\section{REFERENCES}

[1] L. Auslander, I. C. Gertner and R. Tolimieri, "The discrete Zak transform application to time-frequency analysis and synthesis of nonstationary signals," IEEE Trans. Signal Processing, vol. 39, pp. 825-835, 1991.

[2] M. J. Bastiaans, "Gabor's signal expansion of a signal into Gaussian elementary signals," Proc. IEEE, vol. 68, pp. 538-539, 1980.

[3] _ _A sampling theorem for the complex spectrogram, and Gabor's expansion of a signal in Gaussian elementary signals," Opt. Eng., vol. 20, no. 4, pp. 594-598, 1981.

[4] "Gabor's signal expansion and its relation to sampling of the sliding-window spectrum," Adv. Topics Shannon Sampling Interpolation Theory, R. J. Marks, II, Ed., pp. 1-35.

[5] J. J. Benedetto and D. F. Walnut, "Gabor frames for $L^{2}$ and related spaces," Wavelets Math. Applications, J. Benedetto and M. Frazier, Eds., 1993, pp. 97-162.

[6] J. J. Benedetto, C. Heil, and D. F. Walnut, "Differentiation and Balianlow theorem," J. Fourier Anal. Applicat., vol. 1, pp. 355-402, 1995.

[7] I. Daubechies, Ten Lectures on Wavelets. Philadelphia, PA: SIAM, 1992.

[8] — "The wavelet transform, time-frequency localization, and signal analysis," IEEE Trans. Inform. Theory, vol. 36, pp. 961-1005, 1990.

[9] T. Ebrahimi and M. Kunt, "Image compression by Gabor expansion," Opt. Eng., vol. 30, pp. 873-880, 1991.

[10] H. G. Feichtinger and O. Christensen, "New efficient methods for Gabor analysis," in Proc. SPIE Visual Commun. Image Process., B. G. Haskell and H.-M. Hang, Eds., vol. 2094, pp. 987-998, 1993.

[11] H. G. Feichtinger and K. Gröchenig, "Gabor wavelets and the Heisenberg Groups: Gabor expansions and short time Fourier transform from the group theoretical point of view," Wavelets Tutorial Theory Applications, C. K. Chui, Ed., pp. 359-397, 1992.

[12] D. Gabor, "Theory of communication," J. Elect. Eng., vol. 93, pp. 429-441, 1946.

[13] C. E. Heil and D. F. Walnut, "Continuous and discrete wavelet transforms," SIAM Rev., vol. 31, no. 4, pp. 628-666, 1989.

[14] R. A. Horn and C. A. Johnson, Matrix Analysis. New York: Cambridge Univ. Press, 1988.

[15] E. Isaacson and H. B. Keller, Analysis for Numerical Methods. New York: Wiley, 1966 
[16] R. S. Orr, "Derivation of the finite discrete Gabor transform by periodization and sampling," Signal Process., vol. 34, pp. 85-97, 1993.

[17] _ "The order of computation for finite discrete Gabor transform," IEEE Trans. Signal Processing, vol. 41, pp. 122-130, Feb. 1993.

[18] M. Porat and Y. Y. Zeevi, "The generalized Gabor scheme of image representation in biological and machine vision," IEEE Trans. Pattern Anal. Machine Intell., vol. 10, pp. 452-468, 1988.

[19] S. Qian and D. Chen, "Discrete Gabor transform," IEEE Trans. Signal Processing, vol. 41, pp. 2429-2438, 1993.

[20] "Optimal biorthogonal analysis window function for discrete Gabor transform," IEEE Trans. Signal Processing, vol. 42, pp. 694-697, 1994.

[21] S. Qiu, "Gabor transform with undersampling," in Proc. IEEE-SP Int. Symp. Time-Frequency Time-Scale Anal., 1996, pp. 317-320.

[22] S. Qiu and H. G. Feichtinger, "Discrete Gabor structure and optimal representation," IEEE Trans. Signal Processing, vol. 43, pp. 2258-2268, Oct. 1995.

[23] S. Qiu, "Block-circulant Gabor-matrix structure and Gabor transforms," Opt. Eng., vol. 34, pp. 2872-2878, 1995.

[24] D. Stewart, L. Potter, and S. C. Ahalt, "Computationally attractive real Gabor transform," IEEE Trans. Signal Processing, vol. 43, pp. 77-84, Jan. 1995.

[25] G. Strang, "The fundamental theorem of linear algebra," Amer. Math. Monthly, vol. 100, no. 9, pp. 848-855, 1993.

[26] G. Strang, Linear Algebra and its Applications, 2nd ed. New York: Academic, 1980.
[27] R. Tolimieri and R. S. Orr, "Poisson summation, the ambiguity function and the rheory of Weyl-Heisenberg frames," J. Fourier Anal. Applicat., vol. 1, pp. 233-248, 1995.

[28] J. Wexler and S. Raz, "Discrete Gabor expansion," Signal Process., vol. 21, pp. 207-220, 1990.

[29] J. Yao, "Complete Gabor transformation for signal representation," IEEE Trans. Image Processing, vol. 2, pp. 152-159, Apr. 1993.

[30] J. Yao, P. Krolak, and C. Steele, "The generalized Gabor transform," IEEE Trans. Image Processing, vol. 4, pp. 978-988, July 1995.

[31] M. Zibulski and Y. Y. Zeevi, "Oversampling in the Gabor scheme," IEEE Trans. Signal Processing, vol. 41, pp. 2670-2687, Aug. 1993.

Sigang Qiu received the Master's degree in mathematics from Qufu Normal University, Qufu, China, and the M.S. degree in applied mathematics from the University of Connecticut, Storrs, in 1989 and 1996, respectively. He is scheduled to receive the Ph.D. degree from the University of Vienna, Vienna, Austria, in 1998.

He is currently a Digital Signal Processing Engineer at Cirrus Logic, Raleigh, NC, where he has worked on modern designs since August 1997. His current interests are in the areas of communication and signal processing. He has worked extensively on numerical analysis, Fourier analysis and Gabor theory, and wavelets and their application in engineering. 